\title{
Asymptotical stability of Runge-Kutta for a class of impulsive differential equations
}

\section{Gui-Lai Zhang*}

"Correspondence: guilaizhang@126.com

School of Mathematics and

Statistics, Northeastern University at

Qinhuangdao, Qinhuangdao, Hebei 066004, China

\begin{abstract}
The aim of this paper is to study asymptotical stability of Runge-Kutta methods for a class of linear impulsive differential equations with piecewise continuous arguments. New results about the asymptotical stability region of Runge-Kutta methods for these equations are obtained by the theory of the Padé approximation. Finally, some numerical examples are given to illustrate the theoretical results.
\end{abstract}

Keywords: asymptotical stability; Runge-Kutta methods; impulsive differential equations; piecewise constant arguments; stability region

\section{Introduction}

In the past two decades, the theory of impulsive differential equations has been developed rapidly [1-3]. Such equations consist of differential equations with impulse effects and emerge in modeling of real-world problems observed in engineering, physics, biology, etc. In addition to these, the theory of numerical methods for impulsive differential equations has also been studied extensively [4-7].

In 1984, Cooke and Wiener studied differential equations without impulses and they noted that such equations were comprehensively related to impulsive and difference equations [8]. Later, the case of discontinuous solutions of differential equations with piecewise continuous arguments was proposed as an open problem by Wiener [9]. On the other hand, it is well known that many biological phenomena involving thresholds, bursting rhythm models in medicine and biology, and optimal control models in economics do exhibit impulsive effects [3]. Recently, the existence and uniqueness, and oscillation of the exactly solutions of impulsive delay differential equations with piecewise constant arguments have been widely studied [10-13]. But to the best of our knowledge, up to now, there are few articles referring to numerical methods for impulsive delay differential equations with piecewise constant arguments.

In this paper, we study the asymptotical stability of Runge-Kutta methods for the following scalar equation:

$$
\begin{cases}x^{\prime}(t)=p x(t)+q x([t]), & t \geq 0, t \neq k, k=1,2, \ldots, \\ \Delta x(k)=r x\left(k^{-}\right), & k=1,2, \ldots, \\ x(0)=x_{0}, & \end{cases}
$$


where $p, q, r$, and $x_{0}$ are real constants and [.] denotes the greatest integer function. This paper can be seen not only as the extension of asymptotically stable theory of differential equations with piecewise arguments in [14], but also as the extension of asymptotically stable theory of impulsive ordinary differential equations in [4].

The paper is organized as follows. In Section 2, we obtain the existence, uniqueness, and asymptotical stability of the exact solutions of (1.1). In Section 3, we study the asymptotical stability of the Runge-Kutta methods for (1.1). In Section 4, two special cases of Section 3 are studied, respectively: impulsive differential equations without piecewise constant argument and differential equations with piecewise constant arguments. In Section 5, some numerical examples are given to confirm the theoretical results.

\section{Asymptotical stability of the exact solutions}

Definition 2.1 (See [10-12,15]) If a function $x: \mathbb{R}^{+} \rightarrow \mathbb{R}$ satisfies the following conditions, it is said to be a solution of (1.1):

(1) $x(t)$ is continuous for $t \in[0,+\infty)$ with the possible exception of the points $[t] \in[0, \infty)$,

(2) $x(t)$ is right continuous and has left-hand limit at the points $[t] \in[0, \infty)$,

(3) $x(t)$ is differentiable and satisfies $x^{\prime}(t)=p x(t)+q x([t])$ for any $t \in \mathbb{R}^{+}$with the possible exception of the points $[t] \in[0, \infty)$ where one-sided derivatives exist,

(4) $x(n)$ satisfies $\Delta x(n)=r x\left(n^{-}\right)$for $n \in \mathbb{Z}^{+}$.

Definition 2.2 The zero solution of (1.1) is said to be asymptotically stable, if

$$
\lim _{t \rightarrow \infty} x(t)=0
$$

where $x(t)$ is the solution of (1.1) for any initial data $x_{0}$.

By [15], p.179, Theorem 3, and [15], p.183, Theorem 9, we immediately obtain the following theorem.

Theorem 2.3 When $p \neq 0$, on $t \in[0, \infty),(1.1)$ has a unique solution

$$
x(t)=m_{0}(\{t\}) \cdot n_{0}^{[t]} \cdot x_{0},
$$

where $\{t\}$ is the fractional part of $t$ and

$$
m_{0}(t)=\mathrm{e}^{p t}+\frac{q}{p}\left(\mathrm{e}^{p t}-1\right), \quad n_{0}=(1+r) m_{0}(1)=(1+r)\left(\mathrm{e}^{p}+\frac{q}{p}\left(\mathrm{e}^{p}-1\right)\right) .
$$

When $p=0$, on $t \in[0, \infty),(1.1)$ also has a unique solution

$$
x(t)=(1+r)^{[t]}(1+q)^{[t]}(q\{t\}+1) x_{0} .
$$

Consequently, when $p \neq 0$, the zero solution of (1.1) is asymptotically stable if and only if

$$
\left|(1+r)\left(\mathrm{e}^{p}+\frac{q}{p}\left(\mathrm{e}^{p}-1\right)\right)\right|<1
$$


when $p=0$, the zero solution of (1.1) is asymptotically stable if and only if

$$
|(1+r)(1+q)|<1
$$

We introduce the sets $H_{0}$ and $H$ consisting of $(p, q, r) \in \mathbb{R}^{3}$ which satisfy the condition (2.2) and (2.3), respectively, i.e.

$$
\begin{aligned}
& H_{0}=\{(0, q, r):|(1+r)(1+q)|<1\}, \\
& H=\left\{(p, q, r): p \neq 0 \text { and }\left|(1+r)\left(\mathrm{e}^{p}+\frac{q}{p}\left(\mathrm{e}^{p}-1\right)\right)\right|<1\right\},
\end{aligned}
$$

and we divide the region $H$ into four parts as $p+q \neq 0$ :

$$
\begin{aligned}
& H_{1}=\left\{(p, q, r): p<0, \mathrm{e}^{p}<\frac{q}{p+q} \text { and }(p, q, r) \in H\right\}, \\
& H_{2}=\left\{(p, q, r): p<0, \mathrm{e}^{p}>\frac{q}{p+q} \text { and }(p, q, r) \in H\right\}, \\
& H_{3}=\left\{(p, q, r): p>0, \mathrm{e}^{p}<\frac{q}{p+q} \text { and }(p, q, r) \in H\right\}, \\
& H_{4}=\left\{(p, q, r): p>0, \mathrm{e}^{p}>\frac{q}{p+q} \text { and }(p, q, r) \in H\right\} .
\end{aligned}
$$

\section{Runge-Kutta methods for (1.1)}

Consider the Runge-Kutta methods for equation (1.1):

$$
\begin{cases}x_{k, l+1}=x_{k, l}+h \sum_{i=1}^{v} b_{i}\left(p Y_{k, l}^{i}+q x_{k, 0}\right), & l=0, \ldots, m-1, k=0,1, \ldots \\ Y_{k, l}^{i}=x_{k, l}+h \sum_{j=1}^{v} a_{i j}\left(p Y_{k, l}^{j}+q x_{k, 0}\right), & l=0, \ldots, m-1, k=0,1, \ldots \\ x_{(k+1), 0}=(1+r) x_{k, m}, & k=0,1,2, \ldots \\ x_{0,0}=x_{0}, & \end{cases}
$$

where $h=\frac{\tau}{m}, m \geq 1, m$ is an integer, $h$ is the stepsize, $v$ is referred to as the number of stages. The weights are $b_{i}$, the abscissas are $c_{i}=\sum_{j=1}^{v} a_{i j}$ and the matrix $A=\left[a_{i, j}\right]_{i, j=1}^{v}$ will be denoted by $(A, b, c)$.

Definition 3.1 Process (3.1) for equation (1.1) is called asymptotically stable at $(p, q, r)$ if and only if for all $m \geq M$ and $h=\frac{1}{m}$

1. $I-z A$ is invertible where $z=h p$,

2. for any given $x_{k, l}(0 \leq l \leq m)$ by relationship (3.1), such that $\lim _{k \rightarrow \infty} X_{k}=0$ where

$$
X_{k}=\left(x_{k, 0}, x_{k, 1}, \ldots, x_{k, m}\right) \text {. }
$$

Definition 3.2 The set of all pairs $(p, q, r)$ at the process (3.1) for equation (1.1) which is asymptotically stable is called stability region denoted by $S$.

Theorem 3.3 When $p \neq 0$, the method (3.1) is asymptotically stable if and only if

$$
\left|(1+r)\left(R(z)^{m}+\frac{q}{p}\left(R(z)^{m}-1\right)\right)\right|<1,
$$


where $R(z)=1+z b^{T}(I-z A)^{-1} e, e=(1,1, \ldots, 1)^{T}$ is a vector of $v$ dimension. On the other hand, when $p=0$, the consistent Runge-Kutta method (3.1) is asymptotically stable if and only if

$$
|(1+r)(1+q)|<1
$$

Proof The Runge-Kutta method (3.1) can be written as

$$
\left\{\begin{array}{l}
x_{k, l+1}=R(z) x_{k, l}+\frac{q}{p}(R(z)-1) x_{k, 0}, \quad l=1,2, \ldots, m-1, \\
x_{k+1,0}=(1+r) x_{k, m}, \quad k=0,1,2, \ldots
\end{array}\right.
$$

which implies that

$$
\left\{\begin{array}{l}
x_{k, l}=\left(R(z)^{l}+\frac{q}{p}\left(R(z)^{l}-1\right)\right) x_{k, 0}, \quad l=1,2, \ldots, m, \\
x_{k+1,0}=(1+r) x_{k, m}, \quad k=0,1,2, \ldots,
\end{array}\right.
$$

which also implies that

$$
x_{k, l}=\left((1+r)\left(R(z)^{m}+\frac{q}{p}\left(R(z)^{m}-1\right)\right)\right)^{k}\left(R(z)^{l}+\frac{q}{p}\left(R(z)^{l}-1\right)\right) x_{0,0} .
$$

Hence when $p \neq 0$, the method (3.1) is asymptotically stable if and only if $\mid(1+r)\left(R(z)^{m}+\right.$ $\left.\frac{q}{p}\left(R(z)^{m}-1\right)\right) \mid<1$.

On the other hand, the Runge-Kutta method being consistent implies $\sum_{i=1}^{v} b_{i}=1$, which also implies

$$
x_{k, l}=((1+r)(1+q))^{k}(1+l h q) x_{0,0}, \quad l=1,2, \ldots, m-1, k=0,1, \ldots
$$

Consequently, when $p=0$, the consistent Runge-Kutta method (3.1) is asymptotically stable if and only if

$$
|(1+r)(1+q)|<1
$$

Lemma 3.4 If the same Runge-Kutta method applied to the following test equation:

$$
\left\{\begin{array}{l}
y^{\prime}(t)=p y(t), \quad t \geq 0 \\
y(0)=y_{0}
\end{array}\right.
$$

is convergent (in the following, we always assume this condition holds), then we have $\lim _{m \rightarrow \infty, m h=1} R(z)^{m}=\lim _{m \rightarrow \infty, m h=1} R(h p)^{m}=\mathrm{e}^{p}$, where $R(z)=1+z b^{T}(I-z A)^{-1}$ e is the stability function.

Proof Obviously, $y_{1}=R(z) y_{0}$. Step by step, $y_{m}=R(z)^{m} y_{0}$ is an approximation of $y(1)$. Solving equation (3.5), we obtain $y(1)=\mathrm{e}^{p} y_{0}$. Because the method is convergent, we have $\lim _{h \rightarrow 0, m h=1} y_{m}=y(1)$, which implies $\lim _{m \rightarrow \infty, m h=1} R(z)^{m}=\mathrm{e}^{p}$. 
Lemma 3.5 (See [16-19]) The (j,k)-Padé approximation to $\mathrm{e}^{z}$ is given by

$$
R(z)=\frac{P_{j}(z)}{Q_{k}(z)}
$$

where

$$
\begin{aligned}
& P_{j}(z)=1+\frac{j}{j+k} \cdot z+\frac{j(j-1)}{(j+k)(j+k-1)} \cdot \frac{z^{2}}{2 !}+\cdots+\frac{j ! k !}{(j+k) !} \cdot \frac{z^{j}}{j !}, \\
& Q_{k}(z)=1-\frac{k}{j+k} \cdot z+\frac{k(k-1)}{(j+k)(j+k-1)} \cdot \frac{z^{2}}{2 !}+\cdots+(-1)^{k} \cdot \frac{k ! j !}{(j+k) !} \cdot \frac{z^{k}}{k !},
\end{aligned}
$$

with error

$$
\mathrm{e}^{z}-R(z)=(-1)^{k} \cdot \frac{j ! k !}{(j+k) !(j+k+1) !} \cdot z^{j+k+1}+O\left(z^{j+k+2}\right) .
$$

It is the unique rational approximation to $\mathrm{e}^{z}$ of order $j+k$, such that the degrees of numerator and denominator are $j$ and $k$, respectively.

It is easy to prove the following lemma. Therefore, the proof is omitted.

Lemma 3.6 Assume $p \neq 0, p+q \neq 0$, and $f(x)=\left|x+\frac{q}{p}(x-1)\right|$. Then $f(x)$ is decreasing for $x<\frac{q}{p+q}$. On the other hand, $f(x)$ is increasing for $x>\frac{q}{p+q}$.

The set $S$ of all $(p, q, r)$ at which the scheme (3.1) is asymptotically stable is called the asymptotical stability region,

$$
S=\left\{(p, q, r):\left|(1+r)\left(R(z)^{m}+\frac{q}{p}\left(R(z)^{m}-1\right)\right)\right|<1\right\} .
$$

Theorem 3.7 Assume $p \neq 0, p+q \neq 0$, and the stability function of the Runge-Kutta method $R(z)$ is the $(j, k)$-Padé approximation to $\mathrm{e}^{z}, z=h p$. Then for $h=\frac{1}{m}, m$ being an integer,

1. when $h \leq \min \left\{h_{1},-\frac{\varsigma}{p}\right\}, H_{1} \subseteq S$ if and only if $j$ is even,

2. when $h \leq h_{2}, H_{2} \subseteq S$ if and only if $j$ is odd,

3. when $h \leq \min \left\{h_{1}, \frac{\eta}{p}\right\}, H_{3} \subseteq S$ if and only if $k$ is odd,

4. when $h \leq h_{2}, H_{4} \subseteq S$ if and only if $k$ is even,

where $h_{1}=\sup \left\{x: R(h p)^{\frac{1}{h}}<\frac{q}{p+q}\right.$ for all $\left.h \in(0, x)\right\}, h_{2}=\sup \left\{x: R(h p)^{\frac{1}{h}}>\frac{q}{p+q}\right.$ for all $h \in$ $(0, x)\}, \eta$ is a real zero of $Q_{k}(z)$, and $\zeta$ is a real zero of $P_{j}(z)$. (See Table 1.$)$

Proof For brevity, we only prove case 1 of the theorem; the others, which can be proved similarly, are omitted. By Lemma 3.4, we see that $\lim _{m \rightarrow \infty, m h=1} R(z)^{m}=\mathrm{e}^{p} \cdot \mathrm{e}^{p} \leq \frac{q}{p+q}$ implies $R(z)^{m} \leq \frac{q}{p+q}$ for $h \leq h_{1}$. Assume $h \leq \min \left\{h_{1},-\frac{\varsigma}{p}\right\}$.

$\Longleftrightarrow$ ) By Lemma 3.5 and $j$ is even, we can obtain $\mathrm{e}^{z} \leq R(z)$, which implies that $\mathrm{e}^{p} \leq$ $R(z)^{m} \leq \frac{q}{p+q}$ for $m \geq M$. Consequently, by Lemma 3.6, we have

$$
\left|R(z)^{m}+\frac{q}{p}\left(R(z)^{m}-1\right)\right| \leq\left|\mathrm{e}^{p}+\frac{q}{p}\left(\mathrm{e}^{p}-1\right)\right|,
$$

which implies $H_{1} \subseteq S$. 
Table 1 The high order Runge-Kutta methods for (1.1)

\begin{tabular}{lllll}
\hline & Gauss-Legendre & Radau IA, IIA & Lobatto IIIA, IIIB & Lobatto IIIC \\
\hline$(j, k)$ & $(v, v)$ & $(v-1, v)$ & $(v-1, v-1)$ & $(v-2, v)$ \\
$H_{1} \subseteq S$ & $v$ is even & $v$ is odd & $v$ is odd & $v$ is even \\
$H_{2} \subseteq S$ & $v$ is odd & $v$ is even & $v$ is even & $v$ is odd \\
$H_{3} \subseteq S$ & $v$ is odd & $v$ is odd & $v$ is even & $v$ is odd \\
$H_{4} \subseteq S$ & $v$ is even & $v$ is even & $v$ is odd & $v$ is even \\
\hline
\end{tabular}

$\Longrightarrow) H_{1} \subseteq S$ implies $\left|R(z)^{m}+\frac{q}{p}\left(R(z)^{m}-1\right)\right| \leq\left|\mathrm{e}^{p}+\frac{q}{p}\left(\mathrm{e}^{p}-1\right)\right|$. Because $\mathrm{e}^{p} \leq \frac{q}{p+q}, R(z)^{m} \leq$ $\frac{q}{p+q}$, by Lemma 3.6, we obtain $R(z)^{m} \leq \mathrm{e}^{p}$, which implies $R(z) \geq \mathrm{e}^{z}$. By Lemma 3.5, we see that $j$ is even.

\section{Special cases}

In this section, two special cases are studied: the first special case $q=0$, where equation (1.1) is changed as linear impulsive ordinary differential equations; second special case $r=0$, where equation (1.1) is changed as linear differential equations with piecewise continuous argument without impulsive perturbations.

\subsection{Linear impulsive ordinary differential equations}

In this subsection, the special case of (1.1) when $q=0$ is studied. Equation (1.1) is changed as

$$
\begin{cases}x^{\prime}(t)=p x(t), & t \geq 0, t \neq k, k=1,2, \ldots \\ \Delta x(k)=r x\left(k^{-}\right), & k=1,2, \ldots \\ x(0)=x_{0} & \end{cases}
$$

where $p, r$, and $x_{0}$.

Theorem 2.3 is changed to the following result.

Theorem 4.1 On $t \in[0, \infty)$, (4.1) has a unique solution

$$
x(t)=m_{1}(\{t\}) \cdot n_{1}^{[t]} \cdot x_{0},
$$

where $\{t\}$ is the fractional part of $t$ and

$$
m_{1}(t)=\mathrm{e}^{p t}, \quad n_{1}=(1+r) m_{1}(1)=(1+r) \mathrm{e}^{p} .
$$

Consequently, the zero solution of (1.1) is asymptotically stable if and only if

$$
|1+r| \mathrm{e}^{p}<1 .
$$

The asymptotical stability sets $H_{0}, H, H_{2}$, and $H_{4}$ are changed as follows, respectively:

$$
\begin{aligned}
& H_{5}=\{(p, r): p=0 \text { and }|1+r|<1\}, \\
& H_{6}=\left\{(p, r):|1+r| \mathrm{e}^{p}<1\right\}, \\
& H_{7}=\left\{(p, r): p<0 \text { and }(p, r) \in H_{6}\right\},
\end{aligned}
$$


Table 2 The high order Runge-Kutta methods for (4.1)

\begin{tabular}{lllll}
\hline & Gauss-Legendre & Radau IA, IIA & Lobatto IIIA, IIIB & Lobatto IIIC \\
\hline$(j, k)$ & $(v, v)$ & $(v-1, v)$ & $(v-1, v-1)$ & $(v-2, v)$ \\
$H_{7} \subseteq S_{1}$ & $v$ is odd & $v$ is even & $v$ is even & $v$ is odd \\
$H_{8} \subseteq S_{1}$ & $v$ is even & $v$ is even & $v$ is odd & $v$ is even \\
\hline
\end{tabular}

$$
H_{8}=\left\{(p, r): p>0 \text { and }(p, r) \in H_{6}\right\}
$$

Obviously, $H_{1}$ and $H_{3}$ are empty as $q=0$, so it is not considered.

We also consider the Runge-Kutta methods for (4.1):

$$
\begin{cases}x_{k, l+1}=x_{k, l}+h p \sum_{i=1}^{v} b_{i} Y_{k, l}^{i}, & l=0,1, \ldots, m-1, k=0,1, \ldots \\ Y_{k, l}^{i}=x_{k, l}+h p \sum_{j=1}^{v} a_{i j} Y_{k, l}^{j}, & l=0,1, \ldots, m-1, k=0,1, \ldots \\ x_{(k+1), 0}=(1+r) x_{k, m}, & k=0,1,2, \ldots \\ x_{0,0}=x_{0}, & \end{cases}
$$

where $h=\frac{1}{m}, m \geq 1, m$ is an integer, $h$ is the stepsize. The set $S_{1}$ of all $(p, r)$ at which the scheme (4.3) is asymptotically stable is called the asymptotical stability region,

$$
S_{1}=\left\{(p, r):|1+r| \cdot R(z)^{m}<1\right\}
$$

From Theorem 3.7, we immediately obtain the following results.

Theorem 4.2 Assume the stability function for the Runge-Kutta method is $R(z)$, the $(j, k)$ Padé approximation to $\mathrm{e}^{z}, z=p h$. Then for $h=\frac{1}{m}, m$ being an integer,

1. $H_{5} \subseteq S_{1}$ for an arbitrary consistent Runge-Kutta method,

2. when $h \leq h_{3}, H_{7} \subseteq S_{1}$ if and only if $j$ is odd,

3. when $h \leq h_{3}, H_{8} \subseteq S_{1}$ if and only if $k$ is even,

where $h_{3}=\sup \{x: R(h p)>0$ for all $h \in(0, x)\}$. (See Table 2.)

The results obtained in this subsection are consistent with the results Ran et al. in [4].

\subsection{Linear differential equations with piecewise continuous argument}

In this subsection, the special case of (1.1) when $r=0$ is studied. Equation (1.1) is changed as

$$
\left\{\begin{array}{l}
x^{\prime}(t)=p x(t)+q x([t]), \quad t \geq 0, \\
x(0)=x_{0},
\end{array}\right.
$$

where we introduced $p, q$, and $x_{0}$.

Theorem 2.3 is changed to the following result.

Theorem 4.3 On $t \in[0, \infty)$, (4.4) has a unique solution

$$
x(t)=m_{0}(\{t\}) \cdot n_{2}^{[t]} \cdot x_{0},
$$


where $\{t\}$ is the fractional part of $t$ and

$$
m_{0}(t)=\mathrm{e}^{p t}+\frac{q}{p}\left(\mathrm{e}^{p t}-1\right), \quad n_{2}=m_{0}(1)=\mathrm{e}^{p}+\frac{q}{p}\left(\mathrm{e}^{p}-1\right) .
$$

Consequently, when $p \neq 0$, the zero solution of (1.1) is asymptotically stable if and only if

$$
\left|\mathrm{e}^{p}+\frac{q}{p}\left(\mathrm{e}^{p}-1\right)\right|=\mathrm{e}^{p}+\frac{|q|}{p}\left(\mathrm{e}^{p}-1\right)<1 .
$$

Obviously, when $p=0$, the zero solution of (1.1) is asymptotically stable if and only if $|1+q|<1$.

The asymptotical stability sets $H, H_{1}$, and $H_{4}$ are changed as follows, respectively:

$$
\begin{aligned}
& H_{9}=\{(p, q): p=0 \text { and }|q|<1\}, \\
& H_{10}=\left\{(p, q):\left|\mathrm{e}^{p}+\frac{q}{p}\left(\mathrm{e}^{p}-1\right)\right|<1\right\}=\left\{(p, q):-\frac{p\left(\mathrm{e}^{p}+1\right)}{\mathrm{e}^{p}-1}<q<-p\right\}, \\
& H_{11}=\left\{(p, q): p<0 \text { and }(p, q) \in H_{10}\right\}, \\
& H_{12}=\left\{(p, q): p>0 \text { and }(p, q) \in H_{10}\right\} .
\end{aligned}
$$

Obviously, the sets $H_{2}$ and $H_{3}$ are empty when $r=0$, so it is not considered.

We also consider the Runge-Kutta methods for (4.4):

$$
\begin{cases}x_{k, l+1}=x_{k, l}+h \sum_{i=1}^{v} b_{i}\left(p Y_{k, l}^{i}+q x_{k, 0}\right), & l=0,1, \ldots, m-1, k=0,1, \ldots \\ Y_{k, l}^{i}=x_{k, l}+h \sum_{j=1}^{v} a_{i j}\left(p Y_{k, l}^{j}+q x_{k, 0}\right), & l=0,1, \ldots, m-1, k=0,1, \ldots \\ x_{(k+1), 0}=x_{k, m}, & k=0,1,2, \ldots \\ x_{0,0}=x_{0}, & \end{cases}
$$

where $h=\frac{1}{m}, m \geq 1, m$ is an integer, $h$ is the stepsize, $v$ is referred to as the number of stages. The following set $S_{2}$ of all $(p, q)$ at which the scheme (3.1) is asymptotically stable is called the asymptotical stability region,

$$
S_{2}=\left\{(p, q):\left|R(z)^{m}+\frac{q}{p}\left(R(z)^{m}-1\right)\right|<1\right\} .
$$

From Theorem 3.7, we immediately obtain the following results.

Theorem 4.4 Assume the stability function Runge-Kutta method is $R(z)$, the (j,k)-Padé approximation to $\mathrm{e}^{z}, z=p h$. Then for $h=\frac{1}{m}, m$ being an integer,

1. $H_{9} \subseteq S_{2}$ for arbitrary consistent Runge-Kutta method,

2. when $h \leq \min \left\{h_{1},-\frac{5}{p}\right\}, H_{11} \subseteq S_{2}$ if and only if $j$ is even,

3. when $h \leq h_{2}, H_{12} \subseteq S_{2}$ if and only if $k$ is even. (See Table 3.)

The results obtained in this subsection are consistent with the results of Liu et al. in [14]. 
Table 3 The high order Runge-Kutta methods for (4.4)

\begin{tabular}{lllll}
\hline & Gauss-Legendre & Radau IA, IIA & Lobatto IIIA, IIIB & Lobatto IIIC \\
\hline$(j, k)$ & $(v, v)$ & $(v-1, v)$ & $(v-1, v-1)$ & $(v-2, v)$ \\
$H_{11} \subseteq S_{2}$ & $v$ is even & $v$ is odd & $v$ is odd & $v$ is even \\
$H_{12} \subseteq S_{2}$ & $v$ is even & $v$ is even & $v$ is odd & $v$ is even \\
\hline
\end{tabular}

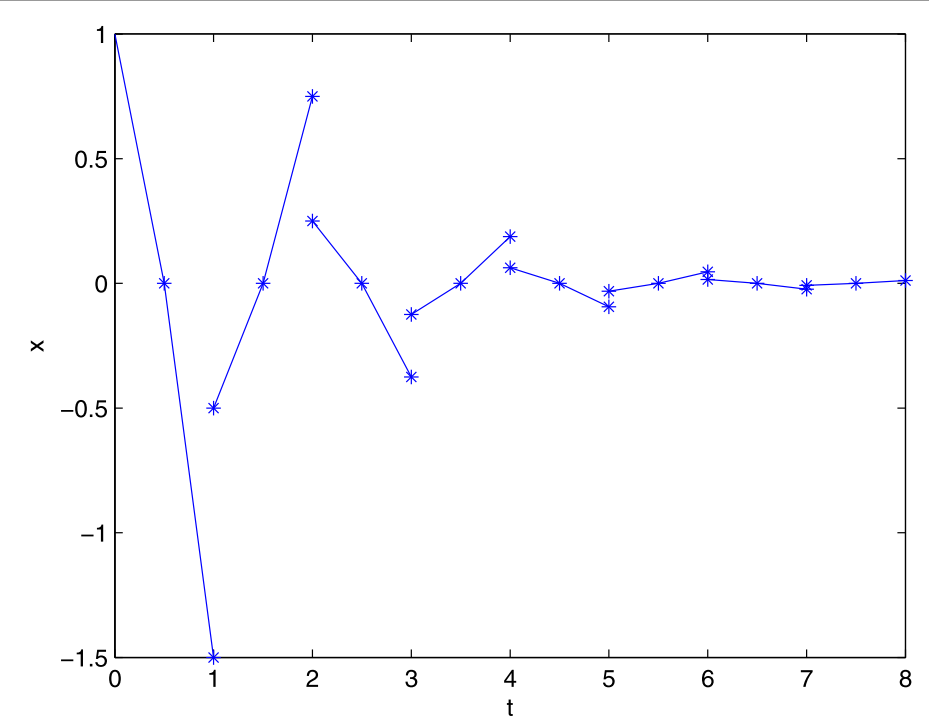

Figure 1 Explicit Euler method for (5.1) as $h=\frac{1}{2}$.

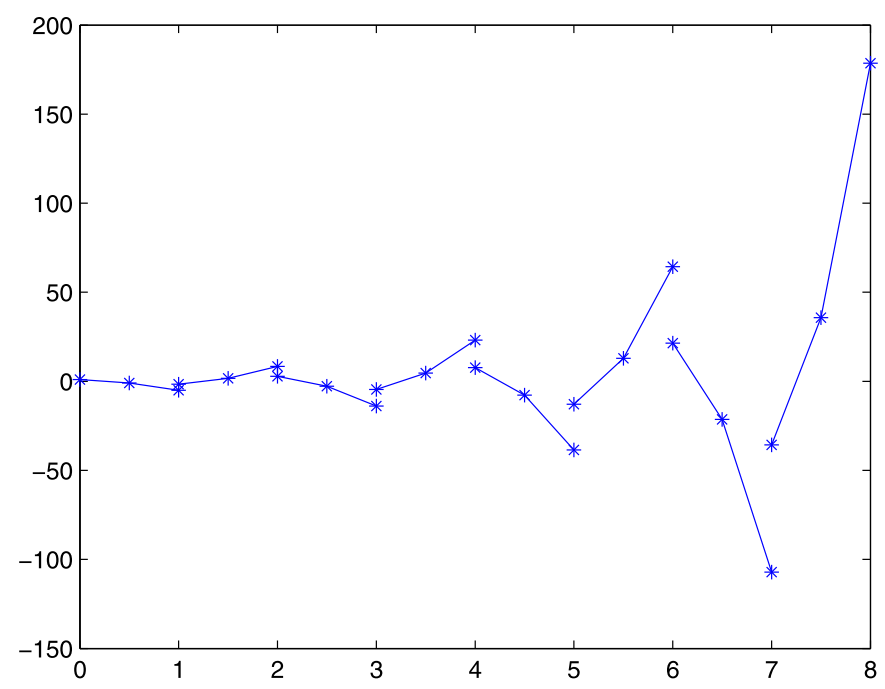

Figure 2 Implicit Euler method for (5.1) as $h=\frac{1}{2}$. 


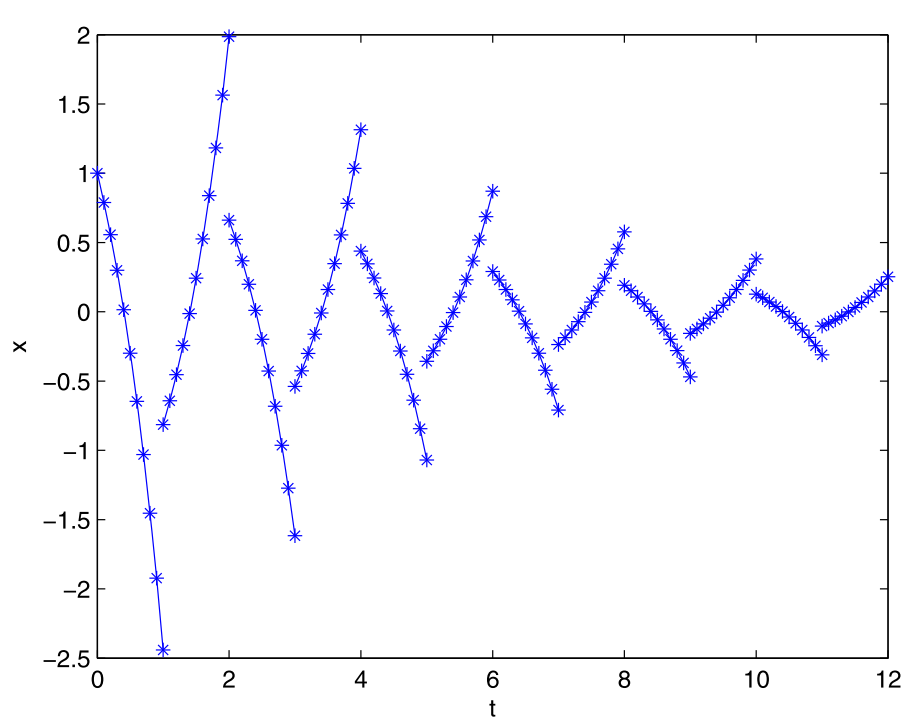

Figure 3 2-stage Lobatto IIIC method for (5.1) as $h=\frac{1}{10}$.

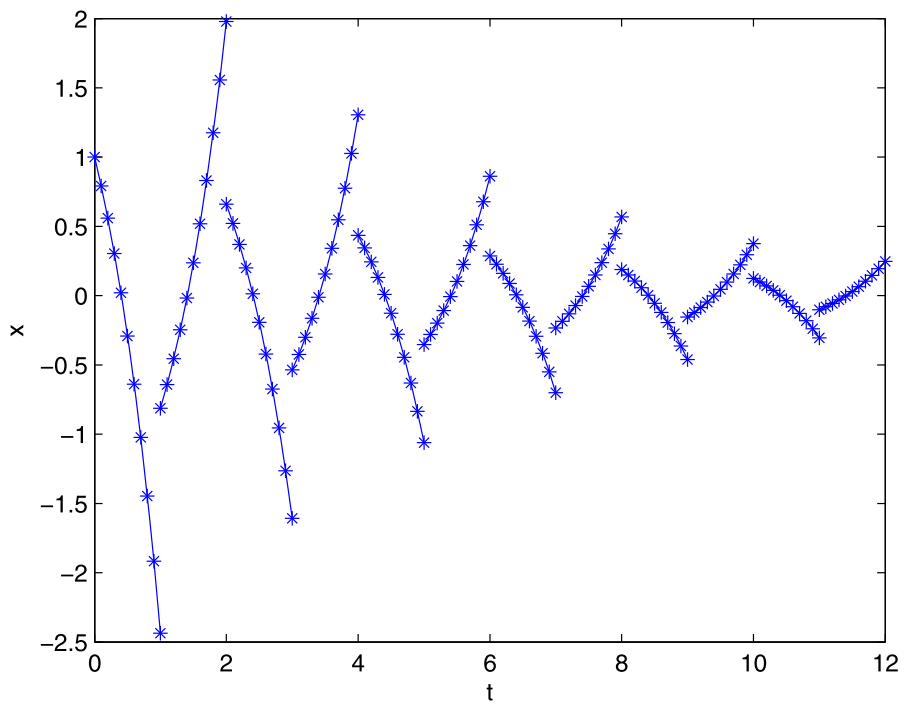

Figure 4 3-stage Lobatto IIIA method for (5.1) as $h=\frac{1}{10}$.

\section{Numerical experiments}

First of all, we consider the following equation:

$$
\begin{cases}x^{\prime}(t)=x(t)-3 x([t]), & t \geq 0, t \neq k, k=1,2, \ldots \\ \Delta x(k)=-\frac{2}{3} x\left(k^{-}\right), & k=1,2, \ldots \\ x(0)=1 & \end{cases}
$$

Obviously, we have $\left|(1+r)\left(\mathrm{e}^{p}+\frac{q}{p}\left(\mathrm{e}^{p}-1\right)\right)\right|<1$. Hence the exact solution of (5.1) is asymptotically stable. 


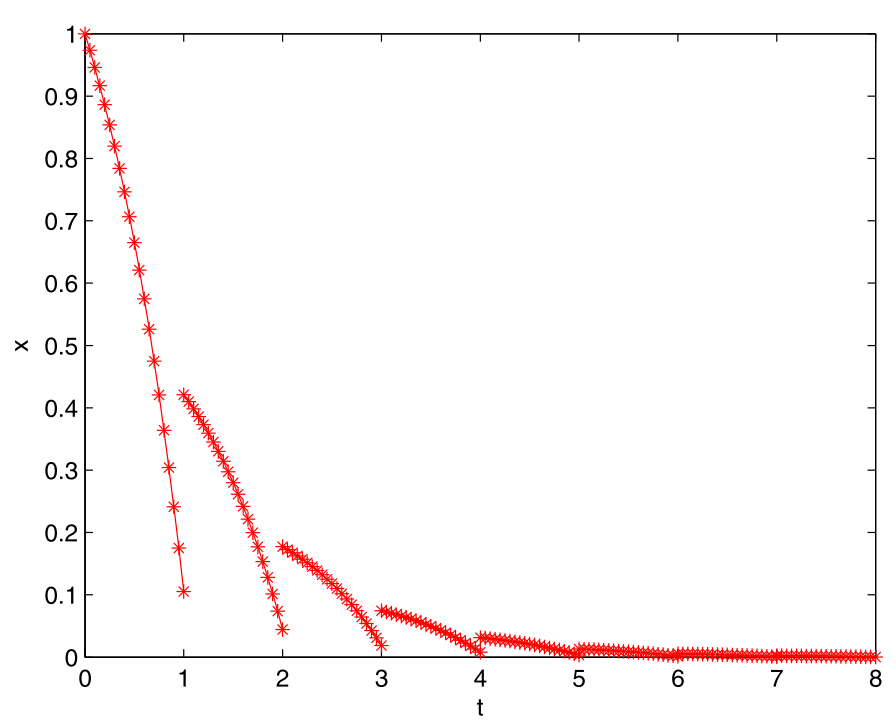

Figure 5 Implicit Euler method for (5.2) as $h=\frac{1}{20}$.

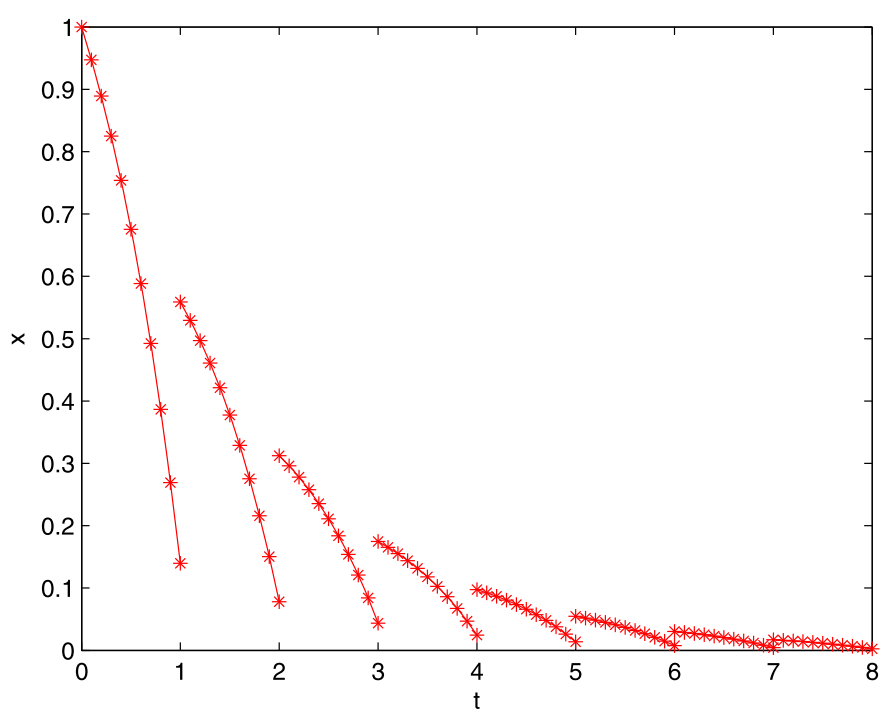

Figure 6 2-stage Lobatto IIIA method for (5.2) as $h=\frac{1}{10}$.

By Theorem 3.7 (case 4 of the theorem), we can see that explicit Euler method for (5.1) (see Figure 1) is asymptotically stable for arbitrary $h=\frac{1}{m}, m$ is an integer. (Obviously, $R(h p)^{\frac{1}{h}}=(1+h p)^{\frac{1}{h}}=\left(1+\frac{1}{m}\right)^{m} \geq 2>\frac{3}{2}=\frac{q}{p+q}$, which implies $h \leq h_{2}$ holds for arbitrary $h=\frac{1}{m}, m$ being an integer.)

We all know that the implicit Euler method for the test equation $y^{\prime}(t)=\lambda y(t), \Re(\lambda)<0$, is asymptotically stable for arbitrary step size $h>0$. But this does not hold for arbitrary step size $h>0$ when the implicit Euler method is adopted for (5.1) (see Figure 2).

By Theorem 3.7 and Table 1, we can also see that the 2-stage Lobatto IIIC method and the 3 -stage Lobatto IIIA method are all asymptotically stable (see Figures 3 and 4 ) for $h$ small enough. 


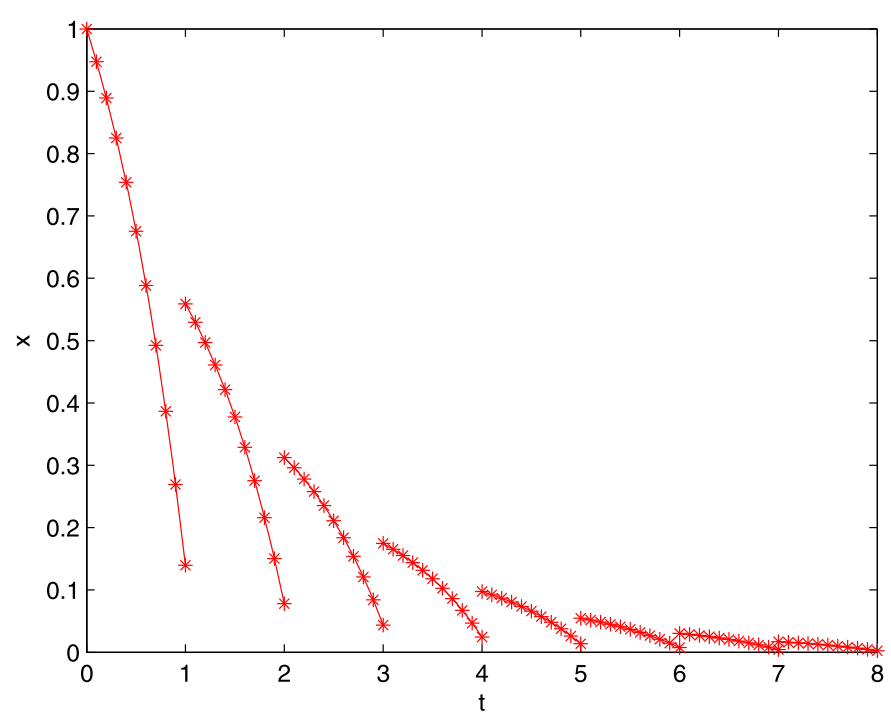

Figure 7 2-stage Lobatto IIIB method for (5.2) as $h=\frac{1}{10}$.

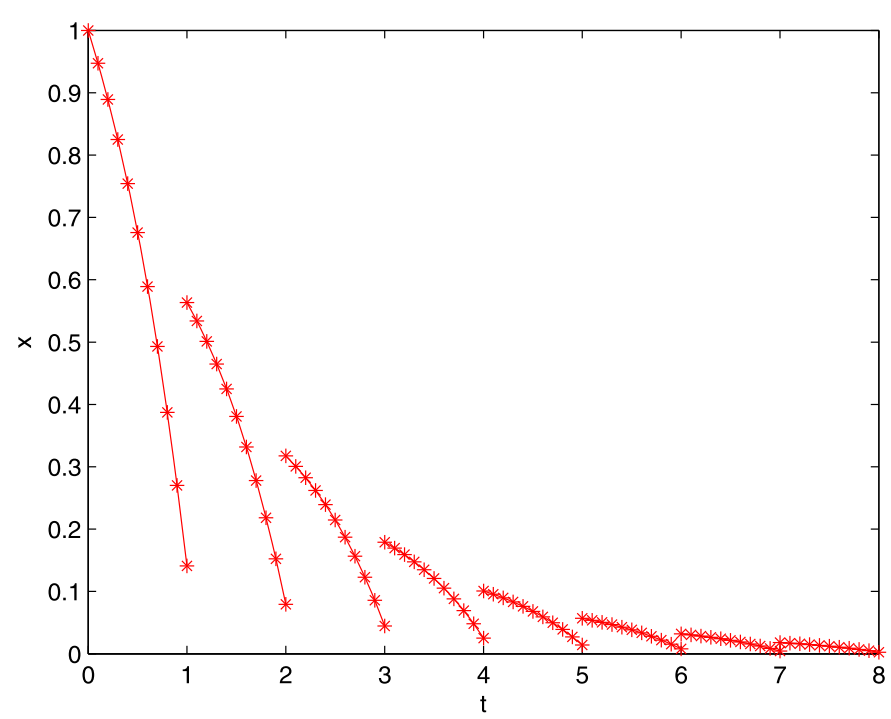

Figure 8 3-stage Lobatto IIIC method for (5.2) as $h=\frac{1}{10}$.

Consider the following equation:

$$
\begin{cases}x^{\prime}(t)=x(t)-\frac{3}{2} x([t]), & t \geq 0, t \neq k, k=1,2, \ldots \\ \Delta x(k)=3 x\left(k^{-}\right), & k=1,2, \ldots \\ x(0)=1 & \end{cases}
$$

Obviously, we have $\left|(1+r)\left(\mathrm{e}^{p}+\frac{q}{p}\left(\mathrm{e}^{p}-1\right)\right)\right|=2(3-\mathrm{e})<1$. Hence the exact solution of (5.2) is asymptotically stable.

By Theorem 3.7 (case 3 of the theorem), we can also see that the implicit Euler method for (5.1) (see Figure 5) is asymptotically stable for $h=\frac{1}{m}, m$ being an integer and $m \geq 6$. 
Table 4 The errors of the Runge-Kutta methods for (5.1)

\begin{tabular}{|c|c|c|c|c|c|c|}
\hline \multirow[t]{2}{*}{$m$} & \multicolumn{2}{|c|}{ The implicit Euler } & \multicolumn{2}{|c|}{ 2-Lobatto IIIA } & \multicolumn{2}{|l|}{ 2-Lobatto IIIC } \\
\hline & $\overline{\mathrm{AE}}$ & $\mathrm{RE}$ & $\overline{\mathrm{AE}}$ & $\mathrm{RE}$ & $\overline{\mathrm{AE}}$ & $\mathrm{RE}$ \\
\hline 10 & 0.2731 & 2.1864 & 0.0023 & 0.0188 & 0.0049 & 0.0393 \\
\hline 20 & 0.0956 & 0.7651 & 5.8209 & 0.0047 & 0.0012 & 0.0096 \\
\hline 40 & 0.0406 & 0.3251 & $1.4524 \mathrm{e}-004$ & 0.0012 & $2.9546 \mathrm{e}-004$ & 0.0024 \\
\hline 80 & 0.0188 & 0.1504 & 3.6293e-005 & 2.9057e-004 & 7.3237e-005 & $5.8636 \mathrm{e}-004$ \\
\hline 160 & 0.0090 & 0.0724 & $9.0721 \mathrm{e}-006$ & $7.2634 \mathrm{e}-005$ & $1.8227 \mathrm{e}-005$ & $1.4594 \mathrm{e}-004$ \\
\hline 320 & 0.0044 & 0.0355 & $2.2680 \mathrm{e}-006$ & $1.8158 \mathrm{e}-005$ & $4.5464 \mathrm{e}-006$ & $3.6400 e-005$ \\
\hline Ratio & 2.2977 & 2.2977 & 4.0083 & 4.0083 & 4.0435 & 4.0435 \\
\hline
\end{tabular}

Table 5 The errors of the Runge-Kutta methods for (5.2)

\begin{tabular}{|c|c|c|c|c|c|c|}
\hline \multirow[t]{2}{*}{$m$} & \multicolumn{2}{|c|}{ The implicit Euler } & \multicolumn{2}{|c|}{ 2-Lobatto IIIA } & \multicolumn{2}{|l|}{ 2-Lobatto IIIC } \\
\hline & $\overline{\mathrm{AE}}$ & RE & $\overline{\mathrm{AE}}$ & $\mathrm{RE}$ & $\overline{\mathrm{AE}}$ & $\mathrm{RE}$ \\
\hline 100 & 0.0013 & 0.3930 & $2.5918 \mathrm{e}-006$ & $8.0380 \mathrm{e}-004$ & $5.2281 \mathrm{e}-006$ & 0.0016 \\
\hline 200 & $7.0145 e-004$ & 0.2175 & $6.4811 e-007$ & $2.0100 \mathrm{e}-004$ & $1.3014 \mathrm{e}-006$ & $4.0362 \mathrm{e}-004$ \\
\hline 400 & $3.6926 \mathrm{e}-004$ & 0.1145 & $1.6204 \mathrm{e}-007$ & $5.0254 \mathrm{e}-005$ & $3.2470 \mathrm{e}-007$ & $1.0070 \mathrm{e}-004$ \\
\hline 800 & $1.8947 \mathrm{e}-004$ & 0.0588 & $4.0510 \mathrm{e}-008$ & $1.2564 \mathrm{e}-005$ & $8.1098 \mathrm{e}-008$ & $2.5151 \mathrm{e}-005$ \\
\hline 1,600 & $9.5971 \mathrm{e}-005$ & 0.0298 & $1.0128 \mathrm{e}-008$ & $3.1409 \mathrm{e}-006$ & $2.0265 e-008$ & $6.2848 \mathrm{e}-006$ \\
\hline 3,200 & $4.8298 \mathrm{e}-005$ & 0.0150 & $2.5319 \mathrm{e}-009$ & $7.8522 \mathrm{e}-007$ & $5.0649 \mathrm{e}-009$ & $1.5708 \mathrm{e}-006$ \\
\hline Ratio & 1.9326 & 1.9227 & 3.9997 & 3.9997 & 4.0064 & 4.0064 \\
\hline
\end{tabular}

(Obviously, $\frac{\eta}{p}=1$ and $R(h p)^{\frac{1}{h}}=\left(\frac{1}{1-h p}\right)^{\frac{1}{h}}=\left(1-\frac{1}{m}\right)^{-m}<3=\frac{q}{p+q}$, which implies $h \leq \min \left\{h_{1}, \frac{\eta}{p}\right\}$ holds for $h=\frac{1}{m}, m$ being an integer and $m \geq 6$.)

Similarly, we can also see that the 2-stage Lobatto IIIA method for (5.1) (see Figure 6) is asymptotically stable for $h=\frac{1}{m}, m$ being an integer and $m \geq 2$. (Obviously, $\frac{\eta}{p}=2$ and $R(h p)^{\frac{1}{h}}=\left(\frac{1+\frac{h p}{2}}{1-\frac{h p}{2}}\right)^{\frac{1}{h}}=\left(1+\frac{2}{2 m-1}\right)^{m}<3=\frac{q}{p+q}$, which implies $h \leq \min \left\{h_{1}, \frac{\eta}{p}\right\}$ holds for $h=\frac{1}{m}$, $m$ being an integer and $m \geq 2$.)

By Theorem 3.7 and Table 1, we also see that the 2-stage Lobatto IIIB and the 3-stage Lobatto IIIC (see Figures 7 and 8 ) are all asymptotically stable for $h$ small enough.

We can see that the methods conserve their orders of convergence from Table 4 and Table 5 .

\section{Competing interests}

The author declares that they have no competing interests.

\section{Acknowledgements}

The author would like to thank the referees for their helpful comments and suggestions. This work is supported by the Research Fund for Northeastern University at Qinhuangdao XNB201415, the NSF of Hebei Province A2015501130 and Research project of higher school science and technology in Hebei province ZD2015211.

Received: 1 November 2015 Accepted: 27 December 2015 Published online: 03 February 2016

\section{References}

1. Bainov, DD, Simeonov, PS: Impulsive Differential Equations: Periodic Solutions and Applications. Longman Scientific and Technical, Harlow (1993)

2. Samoilenko, AM, Perestyuk, NA: Impulsive Differential Equations. World Scientific, Singapore (1995)

3. Lakshmikantham, V, Bainov, DD, Simeonov, PS: Theory of Impulsive Differential Equations. World Scientific, Singapore (1989)

4. Ran, XJ, Liu, MZ, Zhu, QY: Numerical methods for impulsive differential equation. Math. Comput. Model. 48, 46-55 (2008)

5. Liu, MZ, Liang, H, Yang, ZW: Stability of Runge-Kutta methods in the numerical solution of linear impulsive differential equations. Appl. Math. Comput. 192, 346-357 (2007)

6. Ding, X, Wu, KN, Liu, MZ: The Euler scheme and its convergence for impulsive delay differential equations. Appl. Math. Comput. 216, 1566-1570 (2010)

7. Zhang, ZH, Liang, H: Collocation methods for impulsive differential equations. Appl. Math. Comput. 228, 336-348 (2014) 
8. Cooke, KL, Wiener, J: Retarded differential equations with piecewise constant delays. J. Math. Anal. Appl. 99, 265-297 (1984)

9. Wiener, J: Generalized Solutions of Functional Differential Equations. World Scientific, Singapore (1994)

10. Bereketoglu, H, Oztepe, GS: Asymptotic constancy for impulsive differential equations with piecewise constant argument. Bull. Math. Soc. Sci. Math. Roum. 57, 181-192 (2014)

11. Bereketoglu, H, Oztepe, GS: Convergence of the solution of an impulsive differential equation with piecewise constant arguments. Miskolc Math. Notes 14, 801-815 (2013)

12. Karakoc, F, Bereketoglu, H, Seyhan, G: Oscillatory and periodic solutions of impulsive differential equations with piecewise constant argument. Acta Appl. Math. 110, 499-510 (2010)

13. Karakoc, F, Unal, AO, Bereketoglu, H: Oscillation of nonlinear impulsive differential equations with piecewise constant arguments. Electron. J. Qual. Theory Differ. Equ. 2013, 49 (2013)

14. Liu, MZ, Song, MH, Yang, ZW: Stability of Runge-Kutta methods in the numerical solution of equation $u^{\prime}(t)=a u(t)+a_{0} u([t])$. J. Comput. Appl. Math. 166, 361-370 (2004)

15. Bereketoglu, H, Seyhan, G, Ogun, A: Advanced impulsive differential equations with piecewise constant arguments. Math. Model. Anal. 15, 175-187 (2010)

16. Butcher, JC: The Numerical Analysis of Ordinary Differential Equations: Runge-Kutta and General Linear Methods. Wiley, New York (1987)

17. Dekker, K, Verwer, JG: Stability of Runge-Kutta Methods for Stiff Nonlinear Differential Equations. North-Holland, Amsterdam (1984)

18. Hairer, E, Nørsett, SP, Wanner, G: Solving Ordinary Differential Equations II. Stiff and Differential Algebraic Problems. Springer, Berlin (1993)

19. Wanner, G, Hairer, E, Nørsett, SP: Order stars and stability theorems. BIT Numer. Math. 18, 475-489 (1978)

\section{Submit your manuscript to a SpringerOpen ${ }^{\circ}$ journal and benefit from:}

- Convenient online submission

- Rigorous peer review

- Immediate publication on acceptance

- Open access: articles freely available online

- High visibility within the field

- Retaining the copyright to your article 